\title{
Efficacy and Safety of Use of the Fasting Algorithm for Singaporeans With Type 2 Diabetes (FAST) During Ramadan: A Prospective, Multicenter, Randomized Controlled Trial
}

\author{
Zheng Kang Lum ${ }^{1}$ \\ Zi Rui Kboo \\ Wei Yann See Tob, PharmD, BCPS \\ Sbaikb Abdul Kader Kamaldeen \\ Abdul Shakoor, MBBS, MD, MRCP \\ Keith Yu Kei Tsou, MBBCb, MMed, \\ $M C F P^{4}$ \\ Daniel Ek Kwang Chew, MBBS, \\ MRCP, FAMS ${ }^{3}$ \\ Rinkoo Dalan, MBBS, MRCP, \\ FAMS $^{3}$
}

Sing Cheer Kwek, MBBS, MMed ${ }^{4}$

Noorani Otbman ${ }^{3}$

Joyce Xia Lian

Raden Nurberyany Bte Sunari ${ }^{4}$

Joyce Yu-Chia Lee, PharmD, BCPS, $B C A C P^{1,2}$

'Department of Pharmacy, Faculty of Science, National University of Singapore

${ }^{2}$ Department of Pharmacy, Tan Tock Seng Hospital, Singapore

${ }^{3}$ Department of Endocrinology, Tan Tock Seng Hospital, Singapore

${ }^{4}$ Department of Family Medicine, National University Polyclinics, Singapore

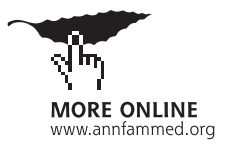

Conflicts of interest: authors report none.

\section{CORRESPONDING AUTHOR}

Joyce Yu-Chia Lee, PharmD, BCPS, BCACP Department of Clinical Pharmacy Practice Susan and Henry Samueli College of Health Sciences

University of California, Irvine

101 Theory, Suite 100

Irvine, CA 92697

j.lee@uci.edu

ORCID iD: 0000-0003-2620-555X

\begin{abstract}
PURPOSE We aimed to evaluate the efficacy and safety of use of the Fasting Algorithm for Singaporeans with Type 2 Diabetes (FAST) during Ramadan.

METHODS We performed a prospective, multicenter, randomized controlled trial. The inclusion criteria were age $\geq 21$ years, baseline glycated hemoglobin $\left(\mathrm{HbA}_{1 \mathrm{c}}\right)$ level $\leq 9.5 \%$, and intention to fast for $\geq 10$ days during Ramadan. Exclusion criteria included baseline estimated glomerular filtration rate $<30 \mathrm{~mL} / \mathrm{min}$, diabetesrelated hospitalization, and short-term corticosteroid therapy. Participants were randomized to intervention (use of FAST) or control (usual care without FAST) groups. Efficacy outcomes were $\mathrm{HbA}_{1 \mathrm{c}}$ level and fasting blood glucose and postprandial glucose changes, and the safety outcome was incidence of major or minor hypoglycemia during the Ramadan period. Glycemic variability and diabetes distress were also investigated. Linear mixed models were constructed to assess changes.
\end{abstract}

RESULTS A total of 97 participants were randomized (intervention: $n=46$, control: $\mathrm{n}=51)$. The $\mathrm{HbA}_{1 \mathrm{c}}$ improvement during Ramadan was 4 times greater in the intervention group $(-0.4 \%)$ than in the control group $(-0.1 \%)(P=.049)$. The mean fasting blood glucose level decreased in the intervention group $(-3.6 \mathrm{mg} /$ $\mathrm{dL})$ and increased in the control group $(+20.9 \mathrm{mg} / \mathrm{dL})(P=.034)$. The mean postprandial glucose level showed greater improvement in the intervention group $(-16.4 \mathrm{mg} / \mathrm{dL})$ compared to the control group $(-2.3 \mathrm{mg} / \mathrm{dL})$. There were more minor hypoglycemic events based on self-monitered blood glucose readings in the control group (intervention: 4, control: 6; $P=.744$ ). Glycemic variability was not significantly different between the 2 groups $(P=.284)$. No between-group differences in diabetes distress were observed $(P=.479)$.

CONCLUSIONS Our findings emphasize the importance of efficacious, safe, and culturally tailored epistemic tools for diabetes management.

Ann Fam Med 2020;18:139-147. https://doi.org/10.1370/afm.2500.

\section{INTRODUCTION}

$\mathrm{T}$ The philosophy of patient empowerment has transformed diabetes care toward a patient-centric model, supporting individuals to be well-informed decision makers responsible for their own health care. Owing to the complex and multifaceted nature of type 2 diabetes as a chronic noncommunicable disease, individuals have to make a lifelong commitment and take an active role in implementing optimal management plans in their everyday life. ${ }^{1}$ Fulfillment of these commitments stems from the balance of intrinsic and extrinsic motivation, which can be influenced by factors at the interpersonal, community, and national levels..$^{2,3}$ For example, social isolation, usually a result of low socioeconomic status or ethnic minority status, can be a barrier to optimal diabetes care. ${ }^{1}$ This might be due to individuals belonging to an ethnic minority group being 
reluctant to seek professional advice on diabetes care because they perceive that health care professionals might not understand their cultural and religious needs. ${ }^{4}$ This was a barrier to optimal self-management and diabetes care established among those of the Muslim community worldwide who fast during Ramadan. ${ }^{4}$ Self-management of type 2 diabetes while fasting during Ramadan can be demanding and challenging for observant Muslim individuals to handle alone, without the support of their health care clinicians. ${ }^{4}$

During Ramadan, observant Muslims abstain from the consumption of food and fluids (including medications) as well as smoking and sexual activities from dawn to dusk. ${ }^{5}$ Sleeping patterns and daily physical activities are also altered during Ramadan. ${ }^{6,7}$ Concerns have been raised regarding the metabolic implications of these behavioral changes, in particular the unpredictability of blood glucose levels in individuals with differing baseline glycemic control. ${ }^{8}$ In addition, the practice of Ramadan fasting increases the risks of complications of acute diabetes, such as hypoglycemia hyperglycemia with or without ketoacidosis, dehydration, and thrombosis, in poorly managed patients. ${ }^{5,9}$ Thus, fasting during Ramadan entails abrupt shifts in meal timing and physical activities, which affect circadian rhythmicity and lifestyle behaviors. ${ }^{5,7,8}$

Although Islamic law exempts the sick from fasting, Ramadan fasting remains a deeply rooted sociocultural practice that provides spiritual enhancement and social cohesion among observant Muslims with type 2 diabetes, with up to $94.2 \%$ reported to fast for at least 15 days during Ramadan. ${ }^{10,11}$ Whereas fasting during Ramadan may promote psychologic and spiritual well-being among the general Muslim population, ${ }^{7}$ several observational studies have revealed struggles among Muslims with diabetes in coordinating self-management requirements, from diet control to medication intake, with the sociocultural demands of Ramadan observance. ${ }^{12,13}$ Ramadan observance presents challenges that can affect psychosocial well-being and compromise self-efficacy in diabetes management, which in turn can contribute to diabetes distress. ${ }^{12,14}$ Diabetes distress refers to negative emotional responses to the stress of coping with the demands of diabetes management and is associated with glycemic control and self-care behavior in individuals with diabetes. ${ }^{14,15}$ The role of health care clinicians in providing technical and emotional support to address diabetes distress cannot be emphasized enough regarding the aim of empowering Muslims who fast to effectively manage their diabetes care during the month of Ramadan. ${ }^{16,17}$

There is no standard practice or culturally tailored epistemic tool to date for the management of type 2 diabetes among Muslims who fast during Ramadan, despite international guidelines made available in recent years. ${ }^{5,10}$ The challenge of universal uptake of the recommendations for multiprong Ramadan diabetes care, targeting patient education and medication management, is global. ${ }^{11}$ In particular, there remains broad variability in health care clinician-led medication modifications. A large-scale multinational study revealed that only $39.3 \%$ of individuals received such support from their health care clinicians during Ramadan. ${ }^{11}$ Little is known in the literature about the role of evidence-based glucose-lowering medication adjustments during the month of Ramadan because such adjustments are generally conducted at the health care clinician's discretion, and they might have differing experience or competency in managing culturally sensitive diabetes care. ${ }^{11}$

To address the need for a culturally tailored standard for diabetes care during the month of Ramadan, a multidisciplinary team of clinicians gathered to design an empowerment-based collaborative clinical tool called the Fasting Algorithm for Singaporeans with Type 2 Diabetes (FAST).$^{18}$ It is a stepwise clinical decision-making tool with risk-assessment screening, Ramadan-specific patient education with self-monitoring of blood glucose (SMBG), structured glucose-lowering medication modification guidance for health care clinicians, and novel self-dose adjustment guidance based on SMBG readings during Ramadan. ${ }^{18}$ The FAST tool aims to guide an effective and safe fasting experience during Ramadan via active clinicianpatient engagement and empowerment of Muslims as informed decision makers in their self-care management during Ramadan by promoting SMBG and relevant medication adjustments. ${ }^{18}$ The primary objective of the present study was to evaluate the efficacy and safety of the FAST tool. Because Ramadan observance may have varying effects on psychologic well-being, especially among people who fast, we also investigated the effects of actions taken by participants via guidance from FAST on diabetes distress.

\section{METHODS}

\section{Trial Design}

We conducted a prospective, multicenter, openlabel, parallel-group, randomized controlled trial (NCT03314246 registered with clinicaltrials.gov) over a period of 2 Ramadan cycles from 2017 to 2018 at a tertiary care endocrinology clinic and a primary outpatient health institution in Singapore. Singapore is a multireligious country, with Islam being the second most common religion. ${ }^{19}$ Fasting during Ramadan is typically observed between 5:30 AM to 7:00 PM, for an 
average of 13.5 hours per day in Singapore. ${ }^{18}$ The trial protocol was approved by the Domain Specific Review Board, a local institutional review board, and was performed in accordance to the Declaration of Helsinki ethical standards and the International Conference on Harmonization Good Clinical Practice guideline. All participants provided written informed consent.

\section{Trial Population}

Muslim individuals aged $\geq 21$ years with diagnosed type 2 diabetes and a baseline glycated hemoglobin $\left(\mathrm{HbA}_{\mathrm{lc}}\right)$ level of $\leq 9.5 \%$ within 3 months before Ramadan and who intended to fast for $\geq 10$ days were eligible for the trial. Individuals with a baseline estimated glomerular filtration rate $<30 \mathrm{~mL} / \mathrm{min}$ within 3 months before Ramadan were excluded. Other exclusion criteria included a history of recurrent hypoglycemia, diabetes-related hospitalization within 1 month before Ramadan, active short-term corticosteroid therapy, pregnancy, inability to communicate independently, and mental incapacitation.

\section{Randomization}

Stratified randomization according to $\mathrm{HbA}_{\mathrm{lc}}$ level was followed by sequential randomization to 2 groups (FAST and control). Participants were randomized with stratification by $\mathrm{HbA}_{1 \mathrm{c}}$ level to 2 groups $\left(\mathrm{HbA}_{1 \mathrm{c}}<7.0 \%\right.$ $[<53 \mathrm{mmol} / \mathrm{mol}]_{i} \mathrm{HbA}_{\mathrm{cc}} 7.0 \%$ to $\leq 9.5 \%[53 \mathrm{mmol} / \mathrm{mol}$ to $\leq 80 \mathrm{mmol} / \mathrm{mol}]$ ) and by type of glucose-lowering regimen to 2 groups (oral hypoglycemic agents; oral hypoglycemic agents and insulin). Participants were then sequentially randomized to the intervention (FAST) or control (usual care without FAST) group in a $1: 1$ ratio.

\section{Trial Procedures}

Muslim individuals with type 2 diabetes who visited the respective health care institutions were screened from the electronic medical records for eligibility. Eligible individuals were approached within 3 months before Ramadan to obtain written informed consent to participate in the trial. Those who provided informed consent were asked to complete a questionnaire comprising questions on demographic, lifestyle, Ramadan practice, and other humanistic parameters, such as diabetes distress, administered by a trained research assistant. Participants were randomized to the intervention or control group after completion of the questionnaire.

Study investigators were trained and advised to follow the FAST tool closely when managing participants in the intervention group. ${ }^{18}$ (The FAST tool is included in the Supplemental Appendix, http://www. AnnFamMed.org/content/18/2/139/suppl/DC1). Study investigators then trained participants in the interven- tion group to use the FAST tool for adjustment of medication doses guided by SMBG during the month of Ramadan. ${ }^{18}$ Telephone hotline support was available during Ramadan for clarification on the use of the FAST tool. Use of the FAST tool concluded after Ramadan, and participants in the intervention group were instructed to revert back to their usual diabetes regimen and routines. All intervention participants were followed up by their health care team within 1 month after the conclusion of Ramadan. Participants in the control group received usual care, which included regular follow-up, ad-hoc visits, and adjustment of medication doses at the discretion of their physician. The FAST tool was not used by physicians or participants in the control group.

All participants were taught to perform SMBG at least 4 times a day during Ramadan (before Sahur, before Iftar, 2 hours after the meal at Iftar, and at any random time of the day) using a glucometer, test strips, and lancets provided by the study team. Sahur (also known as predawn meal) refers to the meal consumed in the morning before fasting, and Iftar refers to the meal at sunset. All participants were also invited to attend an endocrinologist-led pre-Ramadan education workshop conducted in Malay, which addressed Ramadan-specific self-care practices with lifestyle counseling, appropriate glucose monitoring, glucoselowering medication management, and management of acute diabetes complications. The education materials from the workshop were only made available to participants in the intervention group.

\section{Outcomes}

\section{Clinical Efficacy}

The outcome for clinical efficacy was $\mathrm{HbA}_{1 \mathrm{c}}$ level, a surrogate marker for glycemic control, which is commonly used in practice and research relating to Ramadan studies. ${ }^{12,20}$ Although $\mathrm{HbA}_{1 \mathrm{c}}$ level represents a long-term average over a period of approximately 90 days, blood glucose levels within the immediate past 30 days have a greater effect on the 90 -day $\mathrm{HbA}_{1 \mathrm{c}}$ level than the remaining 60 days prior. ${ }^{21}$

Fasting blood glucose (FBG) and postprandial glucose (PPG) measured 2 hours after a meal were also collected and tracked at 3 time points as follows: (1) pre-Ramadan, defined as 3 months before Ramadan, (2) post-Ramadan, defined as 1 month after Ramadan, and (3) 3-month follow-up, defined as 3 months after Ramadan. Glycemic variability, in terms of frequency of glucose fluctuations, was also investigated during Ramadan with the use of SMBG data. ${ }^{22}$ Coefficient of variation $(\% \mathrm{CV})$ values for blood glucose level, measured via $\mathrm{SMBG}$, were calculated for both the intervention and control groups. ${ }^{22}$ 


\section{Safety}

The safety outcome was incidence of major or minor hypoglycemia events experienced during Ramadan. A major hypoglycemia event was defined as any signs or symptoms requiring assistance from another person. ${ }^{23-25} \mathrm{~A}$ minor hypoglycemia event was defined as signs or symptoms precipitated by known or modifiable causes, such as irregular eating habits or increased daily activities, and could be self-managed quickly with or without ingesting fast-acting glucose. ${ }^{23-25}$ Actual incidents of self-reported minor hypoglycemia events were confirmed with a biochemical cutoff for blood glucose level of $<72 \mathrm{mg} / \mathrm{dL}$ obtained with the glucometer. Questionnaires related to hypoglycemia were administered to both groups during the post-Ramadan period to assess incidences of self-reported major and minor hypoglycemia events during Ramadan.

\section{Diabetes Distress}

Diabetes distress was assessed using the 20-item Problem Areas in Diabetes (PAID) instrument, a validated questionnaire in Singapore available in English and Malay languages. ${ }^{26}$ Each item is measured using a 5 -point Likert scale ranging from 0 (not a problem) to 4 (a serious problem) to assess the participants' negative emotions regarding psychosocial domains of diabetes condition and care. The total PAID score is calculated by the sum of all item scores multiplied by a factor of 1.25. Greater scores on a range of 0 to 100 reflect greater emotional distress regarding diabetes. The PAID instrument was administered to both groups during the pre-Ramadan and post-Ramadan periods to track the difference, if any, between the groups before and during Ramadan.

\section{Statistical Analysis}

The efficacy and safety outcomes were assessed using per-protocol analysis of participants who completed the questionnaire and fasted for at least 10 days during the month of Ramadan. Changes in $\mathrm{HbA}_{1 \mathrm{c},}, \mathrm{FBG}$, and PPG between the pre-Ramadan and post-Ramadan interval and post-Ramadan and 3-month follow-up interval were assessed by separate linear mixed models. Random effects modeled were intercepts accounting for variability in participants' glycemic control. Fixed effects modeled were time, group assigned, glucoselowering medication regimen, and number of comorbidities as covariates. The Mann-Whitney $U$ test was used to test for differences in \% CV between the intervention and control groups during Ramadan.

The $\chi^{2}$ test of independence or Fisher exact test was performed to compare between-group differences in major and minor hypoglycemia incidence, as appropriate. Changes in PAID scores were assessed using intent-to-treat analysis. A linear mixed model was created using the same strategy outlined for the efficacy outcome.

The study was powered at $80 \%$ with a 2 -sided type I error of $5 \%$ to detect a within-group difference in $\mathrm{HbA}_{\mathrm{cc}}$ value of $0.5 \%(5.5 \mathrm{mmol} / \mathrm{mol})$ and an $\mathrm{SD}$ of $1.6 \%(17.5 \mathrm{mmol} / \mathrm{mol}){ }^{23}$ The sample size required was determined to be 44 participants per group after accounting for an attrition rate of $20 \%$.

Continuous data are presented as mean (SD) or median (interquartile range), as appropriate. Categoric data are presented as number (percent). All models were adjusted for glucose-lowering regimen and number of comorbidities. Computations were carried out using SPSS version 25.0 (SPSS Inc).

\section{RESULTS}

Of the 138 participants screened for eligibility, 111 were randomized to the intervention $(n=55 ; 49.5 \%)$ and control $(\mathrm{n}=56 ; 50.5 \%)$ groups (Figure 1$)$. Of the 111 participants randomized, 97 were included in the

\section{Figure 1. Flow diagram for trial participants.}

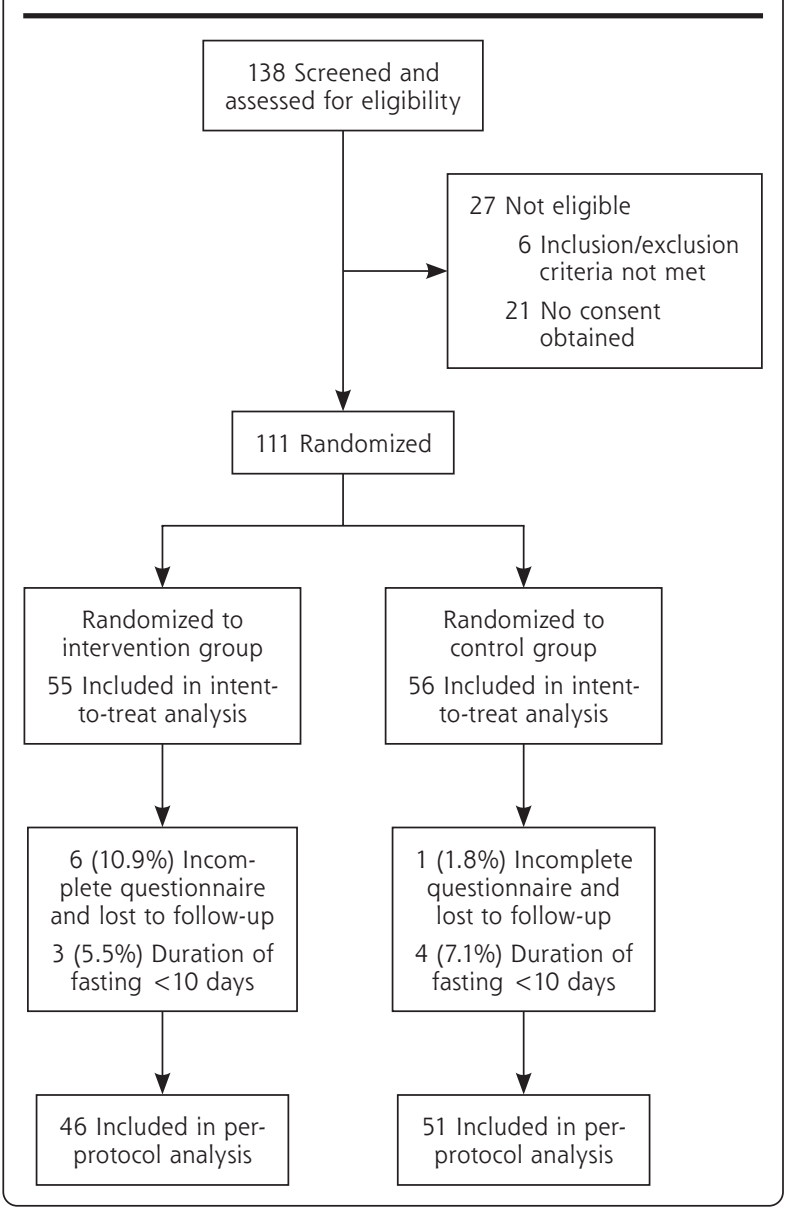


per-protocol analysis, with $46(47.4 \%)$ in the intervention group and $51(52.6 \%)$ in the control group.

Baseline demographic and clinical characteristics (including ethnicity) were similar for both groups, with the exception of sex $(P=.022)$ (Table 1). Overall, participants in both groups fasted for a median of 30 (26-30) days. The mean age was 59.5 (11.2) years, with a median diabetes duration of $10(5-20)$ years. The mean baseline $\mathrm{HbA}_{1 \mathrm{c}}$ level was 7.8 (0.9)\% (62 [9.9] $\mathrm{mmol} / \mathrm{mol}$ ), and the mean baseline FBG and PPG levels were 120.8 (28.9) $\mathrm{mg} / \mathrm{dL}$ and 196.4 (55.9) $\mathrm{mg} / \mathrm{dL}$, respectively.

\section{Clinical Efficacy}

\section{Change in Mean $\mathrm{HbA}_{1 \mathrm{c}}$ Level}

Participants in the intervention group had a significant $(P=.018)$ decrease in $\mathrm{HbA}_{1 \mathrm{c}}$ level of $0.4 \%$ (4.4 mmol/ mol) from the pre-Ramadan to the post-Ramadan timepoint, whereas the $\mathrm{HbA}_{1 \mathrm{c}}$ decrease in the control group was not significant $(P=.202)$ (Figure $2)$. The significant decrease in mean $\mathrm{HbA}_{1 \mathrm{c}}$ level in the intervention group was 4 times that of the control group (intervention: $-0.4 \%[-4.4 \mathrm{mmol} /$ mol] vs control: $-0.1 \%[-1.1 \mathrm{mmol} /$ $\mathrm{mol}]_{;} 95 \% \mathrm{CI},-0.605$ to -0.001 ; $P=.049)$. From the post-Ramadan to the 3-month follow-up timepoint, when participants reverted back to their usual diabetes regimen and routines, no significant difference in change in $\mathrm{HbA}_{1 \mathrm{c}}$ level was observed for either group (intervention: $+0.2 \%$ [2.2 mmol/mol] vs control: $+0 \%[0$ $\mathrm{mmol} / \mathrm{mol}]_{;} 95 \% \mathrm{CI},-0.04$ to 0.769 ; $P=.077)$ (Figure 2).

\section{Change in Mean (SD) FBG and PPG} The mean FBG level for the intervention group decreased from the pre-Ramadan to the post-Ramadan timepoint; the decrease in mean FBG was sustained at the 3-month follow-up (from 123.7 [27.1] mg/ $\mathrm{dL}$ to $122.4[14.5] \mathrm{mg} / \mathrm{dL}$ to 120.1 [27.1] mg/dL) (Figure 3a). However, the mean FBG level for the control group increased from 118.6 (32.5) $\mathrm{mg} / \mathrm{dL}$ (pre-Ramadan) to 134.3 (36.1) $\mathrm{mg} / \mathrm{dL}$ (post-Ramadan) and further increased to $139.5(28.9) \mathrm{mg} / \mathrm{dL}$ at the 3 -month follow-up timepoint.
Changes in the trend of mean FBG levels during the Ramadan period were significantly different between the intervention and control groups (intervention: $-3.6 \mathrm{mg} / \mathrm{dL}$ vs control: $+20.9 \mathrm{mg} / \mathrm{dL}$; between-group difference: $24.5 \mathrm{mg} / \mathrm{dL} ; 95 \% \mathrm{CI}, 11.3-37.7 ; P=.034)$. With the use of FAST, the intervention group managed to maintain mean FBG levels within the range of 80 to $130 \mathrm{mg} / \mathrm{dL}$, as defined by the American Diabetes Association. ${ }^{27}$ In contrast, without the use of FAST, FBG levels in the control group increased beyond the recommended glycemic target range during Ramadan and at the 3-month follow-up.

Overall, changes in the trends of mean PPG levels between the intervention and control groups were not significant $(P=.355)$. However, the direction of PPG

\section{Table 1. Baseline Characteristics of Trial Participants (Per-Protocol Analysis)}

\begin{tabular}{|c|c|c|c|c|}
\hline Characteristic & $\begin{array}{l}\text { Overall } \\
(\mathrm{N}=97)^{\mathrm{a}}\end{array}$ & $\begin{array}{l}\text { Intervention } \\
\text { Group } \\
(n=46)^{a}\end{array}$ & $\begin{array}{l}\text { Control } \\
\text { Group } \\
(n=51)^{a}\end{array}$ & $\begin{array}{c}P \\
\text { Value }\end{array}$ \\
\hline Age, y, mean (SD) & $59.5(11.2)$ & $59.8(10.8)$ & $59.3(11.7)$ & .811 \\
\hline Sex, No. (\%) & & & & .022 \\
\hline Male & $39(40.2)$ & $24(52.2)$ & $15(29.4)$ & \\
\hline Female & $58(59.8)$ & $22(47.8)$ & $36(70.6)$ & \\
\hline Ethnicity, No. (\%) & & & & .440 \\
\hline Malay & $88(90.7)$ & $43(93.5)$ & $45(88.2)$ & \\
\hline Indian & $6(6.2)$ & $2(4.3)$ & $4(7.8)$ & \\
\hline Chinese & $1(1.0)$ & $1(2.2)$ & $0(0)$ & \\
\hline Other & $2(2.1)$ & $0(0)$ & $2(3.9)$ & \\
\hline Education level, No. (\%) & & & & .282 \\
\hline No formal education & $4(4.1)$ & $1(2.2)$ & $3(5.9)$ & \\
\hline Elementary & $29(29.9)$ & $13(28.3)$ & $16(31.4)$ & \\
\hline High school & $43(44.3)$ & $20(43.5)$ & $23(45.1)$ & \\
\hline College/university & $21(21.6)$ & $12(26.1)$ & $9(17.6)$ & \\
\hline $\begin{array}{l}\text { Days fasted during Rama- } \\
\text { dan, median (IQR) }\end{array}$ & $30(26-30)$ & $30(27-30)$ & $30(26-30)$ & .896 \\
\hline $\begin{array}{l}\text { Duration of diabetes, } y_{1} \\
\text { median (IQR) }\end{array}$ & $10(5.0-20.0)$ & $10(5.0-15.0)$ & $11(4.5-21.5)$ & .529 \\
\hline $\begin{array}{l}\text { Number of comorbidities, } \\
\text { mean (SD) }\end{array}$ & $3.75(1.24)$ & $3.72(1.24)$ & $3.78(1.25)$ & .793 \\
\hline Diabetes regimen, No. (\%) & & & & .084 \\
\hline Insulin only & $2(2.1)$ & $2(4.3)$ & $0(0)$ & \\
\hline Oral therapy only & $59(60.8)$ & $31(67.4)$ & $28(54.9)$ & \\
\hline Insulin and oral therapy & $36(37.1)$ & $13(28.3)$ & $23(45.1)$ & \\
\hline BMI, kg/m², mean (SD) & $30.3(5.5)$ & $29.7(5.6)$ & $30.8(5.4)$ & .352 \\
\hline $\mathrm{HbA}_{1 \mathrm{c}}$ mean $(\mathrm{SD})$ & & & & .932 \\
\hline$\%$ & $7.8(0.9)$ & $7.8(0.9)$ & $7.8(0.9)$ & \\
\hline $\mathrm{mmol} / \mathrm{mol}$ & $62(9.9)$ & $62(9.9)$ & $62(9.9)$ & \\
\hline $\begin{array}{l}\text { Fasting blood glucose, } \\
\mathrm{mg} / \mathrm{dL} \text {, mean (SD) }\end{array}$ & $120.8(28.9)$ & $123.7(27.1)$ & $118.6(32.5)$ & .283 \\
\hline $\begin{array}{l}\text { Postprandial glucose, } \\
\text { mg/dL, mean (SD) }\end{array}$ & $196.4(55.9)$ & $191.0(41.5)$ & $201.8(68.5)$ & .867 \\
\hline $\begin{array}{l}\text { Creatinine clearance, } \\
\mathrm{mL} / \mathrm{min} \text {, mean (SD) }\end{array}$ & $60.3(29.6)$ & $58.8(30.1)$ & $61.7(29.4)$ & .635 \\
\hline
\end{tabular}


change differed. For the intervention group, the mean PPG level decreased from $191.0 \mathrm{mg} / \mathrm{dL}$ to the American Diabetes Association's recommended level of $<180 \mathrm{mg} / \mathrm{dL}$ during Ramadan (Figure 3b). For the control group, the mean PPG level fluctuated from $201.8 \mathrm{mg} / \mathrm{dL}$ before Ramadan to $189.2 \mathrm{mg} / \mathrm{dL}$ during Ramadan and to $200.0 \mathrm{mg} /$ $\mathrm{dL}$ at the 3-month follow-up.

The $\% \mathrm{CV}$, measuring glycemic variability, between the intervention and control groups was not significantly different during Ramadan (intervention: $27.2 \%$ vs control: $25.2 \% ; P=.284$ ).

\section{Safety}

There were no self-reported major hypoglycemia events experienced during the Ramadan period for both groups. A total of $14(30.4 \%)$ and $15(29.4 \%)$ incidents of self-reported minor hypoglycemia events during Ramadan were reported by participants in the intervention and control group, respectively (Table 2 ). However, only 1 in the intervention group and 5 in the control group were actual hypoglycemic incidents confirmed by SMBG reading of $<72 \mathrm{mg} / \mathrm{dL}$. Although not statistically significant, the control group had more actual minor hypoglycemic events than the intervention group.

\section{Diabetes Distress}

Improvements in PAID score were observed from the pre-Ramadan to the post-Ramadan timepoint for both groups (intervention: $-8.97, P=.024$ vs control: -10.52 , $P=.006)$. The overall improvement in both groups was similar $(95 \% \mathrm{CI},-2.767$ to $5.859 ; \mathrm{P}=.479)$. Therefore, the use of the FAST tool did not incur additional distress to the participants in the intervention group.

\section{DISCUSSION}

We evaluated the efficacy and safety of the empowerment-based collaborative FAST clinical tool, designed to provide culturally tailored standardized care for Muslims with type 2 diabetes who intend to fast during Ramadan. The novelty of the collaborative FAST clinical tool stemmed from its development framework, which incorporated constructs from established behavioral theories including self-determination theory. ${ }^{18}$ The development of this tool also drew elements from basic physiologic needs theory to target the motivational aspects of optimizing diabetes care during Ramadan. ${ }^{18}$ The FAST tool is a collaborative clinical decision tool that incorporates shared decision making between health care clinicians and fasting individuals and promotes self-efficacy for an effective and safe fasting experience during Ramadan. ${ }^{18}$

Results of the present study showed that use of the FAST tool allowed for greater improvement in glycemic control during Ramadan. Before this study, the effect of Ramadan fasting on glycemic control was found to be affected by support from health care clinicians. ${ }^{23,28}$ By standardizing diabetes care with the FAST tool, intervention participants showed 4 times the amount of improvement in glycemic control $(-0.4 \%$; $-4.4 \mathrm{mmol} / \mathrm{mol}$ ) compared to the control group in terms of change in $\mathrm{HbA}_{1 \mathrm{c}}$ level from pre-Ramadan to post-Ramadan. The control group showed no significant improvement in glycemic control.

In terms of mean FBG change, the intervention group showed a significant improvement compared to the control group from the pre-Ramadan to the postRamadan timepoint, with the improvement sustained at the 3-month follow-up timepoint. These findings support the empowerment characteristics of the FAST tool, such as active SMBG monitoring and self-dose adjustment, which facilitate informed decision making during Ramadan. ${ }^{29}$

The positive effects of empowerment with the FAST tool on glycemic control were mirrored by a systematic review of Ramadan-specific education interventions, in which improvements in $\mathrm{HbA}_{1 \mathrm{c}}$ level were observed (effect size range: $-1.14 \%$ to $+1.70 \%[-12.5$ to $+18.6 \mathrm{mmol} / \mathrm{mol}]){ }^{9}$ The present study also provided evidence for health care clinicians to incorporate cul- 
Figure 3a. Trends of change in mean FBG levels across pre-Ramadan, post-Ramadan, and 3-month follow-up timepoints.

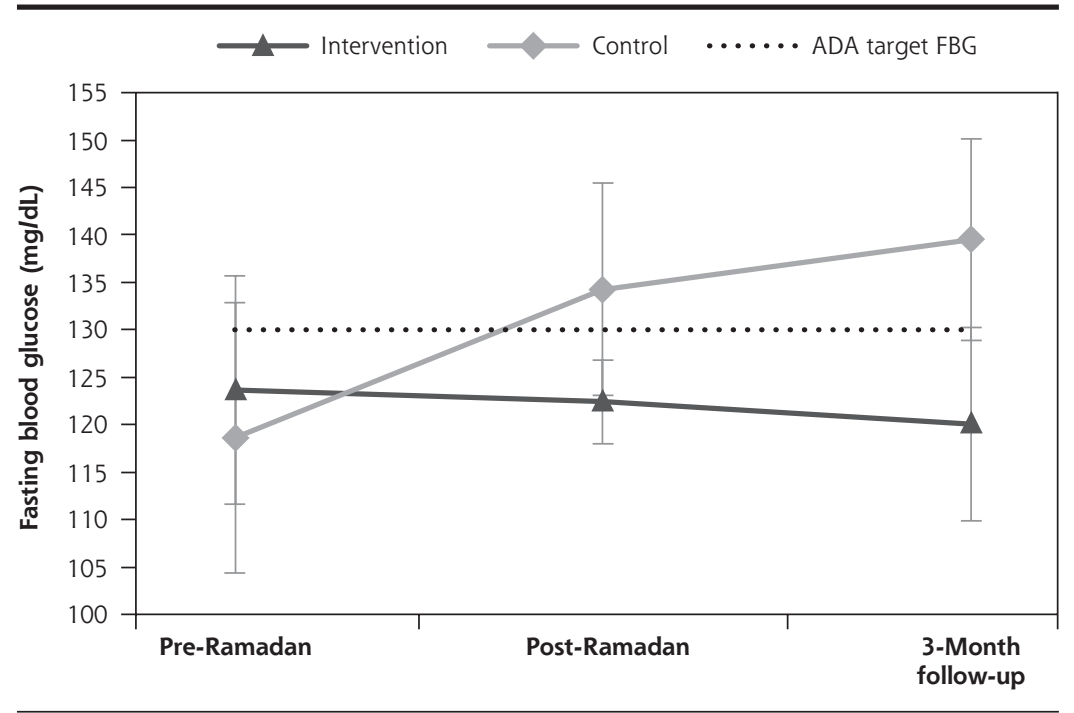

ADA = American Diabetes Association; FBG = fasting blood glucose; PPG = postprandial glucose

Changes in fasting blood glucose during the Ramadan period. The dotted line indicates the target cutoff for fasting blood glucose of $130 \mathrm{mg} / \mathrm{dL}$, as defined by the ADA.

Figure 3b. Trends of change in mean PPG levels across pre-Ramadan, post-Ramadan, and 3-month follow-up timepoints.

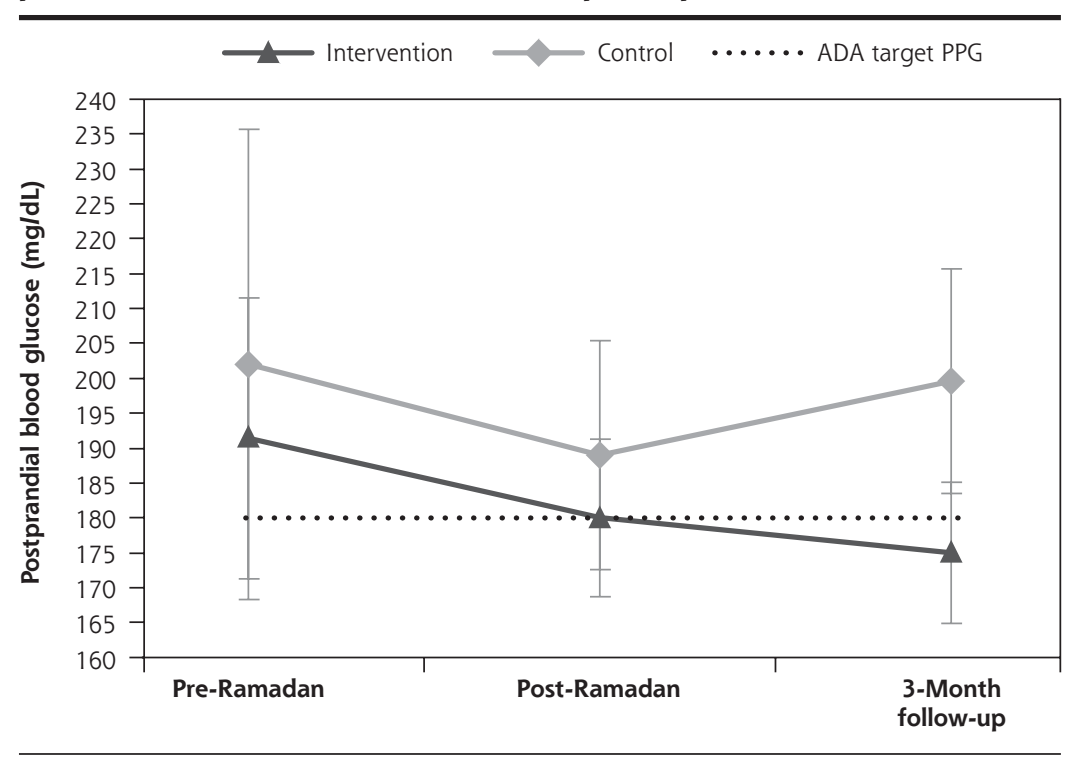

$\mathrm{ADA}=$ American Diabetes Association; FBG = fasting blood glucose; PPG = postprandial glucose

Changes in postprandial glucose during the Ramadan period. The dotted line indicates the target cutoff for postprandial glucose of $180 \mathrm{mg} / \mathrm{dL}$, as defined by the ADA. impaired homeostatic response during Ramadan. ${ }^{5}$ Because the FAST tool was designed to guide clinical decisions during Ramadan, it was not surprising that the $\mathrm{HbA}_{1 \mathrm{c}}$ level reverted to close to baseline when participants returned to their usual diabetes regimen and routines.

The FAST tool also facilitated safe fasting during Ramadan. Hypoglycemia remains a major concern for individuals with type 2 diabetes, owing to the predisposition for hypoglycemia as a result of prolonged fasting. ${ }^{7}$ There were more confirmed incidents of actual minor hypoglycemia during Ramadan in the control group than the intervention group, as shown by objective SMBG findings. This might have clinical implications because hypoglycemia events, whether minor or major, should be minimized to achieve optimal diabetes care. Interestingly, the present study had more unconfirmed self-reported minor hypoglycemia incidents among participants using the FAST tool (intervention: 13; control: 10). These findings suggested that the intervention participants were empowered with enhanced selfawareness of hypoglycemia management via the use of the FAST tool. The present study reaffirms the need for Ramadan-specific education to support individuals with the necessary knowledge and skills to recognize and manage hypoglycemia events for a safe fasting experience. ${ }^{28,30,31}$

Several studies have shown that diabetes distress is directly associated with the behavioral demands of self-care manage- turally tailored modifications in the glucose-lowering regimen during Ramadan. ${ }^{5,18}$ The universal uptake of modifications in glucose-lowering regimen with the use of the FAST tool maintained good glycemic control for individuals with type 2 diabetes. This is important for individuals with type 2 diabetes who may have an ment. ${ }^{17}$ The present study showed that diabetes distress improved significantly from the pre-Ramadan to the post-Ramadan timepoint for both groups, despite the participants having low diabetes distress at baseline. Indeed, the complex nature of diabetes distress can be attributed to multifaceted psychosocial factors dur- 


\section{Table 2. Safety Outcomes}

\begin{tabular}{|c|c|c|c|}
\hline & $\begin{array}{l}\text { Intervention } \\
\text { Group } \\
(n=46)\end{array}$ & $\begin{array}{l}\text { Control } \\
\text { Group } \\
(n=51)\end{array}$ & $P$ Value \\
\hline Self-reported minor hypoglycemia eventa, No. (\%) & $14(30.4)$ & $15(29.4)$ & .912 \\
\hline $\begin{array}{l}\text { Minor hypoglycemia event based solely on SMBG } \\
\text { reading }<72 \mathrm{mg} / \mathrm{dL} \text {, No. (\%) }\end{array}$ & $4(8.7)$ & $6(11.8)$ & .744 \\
\hline Actual minor hypoglycemia event ${ }^{b}$, No. (\%) & $1(2.2)$ & $5(9.8)$ & .154 \\
\hline \multicolumn{4}{|l|}{ SMBG = self-monitoring of blood glucose . } \\
\hline \multicolumn{4}{|c|}{$\begin{array}{l}\text { a Minor hypoglycemia event defined as signs and symptoms precipitated by known or modifiable causes and } \\
\text { could be quickly self-managed with or without ingesting fast-acting glucose. } \\
\text { b Actual minor hypoglycemia event defined as self-reported minor hypoglycemia and a corresponding objec- } \\
\text { tive SMBG reading of }<72 \mathrm{mg} / \mathrm{dL} \text {. }\end{array}$} \\
\hline
\end{tabular}

standardized use of the FAST tool. In addition, a pre-Ramadan education workshop was made available to all participants to streamline the common understanding of diabetes management during Ramadan. Future studies should examine the selfefficacy of fasting individuals during Ramadan with the use of constructs from relevant health behavior models and theories.

In conclusion, we found that the FAST clinical decision tool showed 4 times the efficacy in

ing Ramadan. ${ }^{32}$ The sociocultural aspect of Ramadan observance provides intrapersonal engagement of spiritual well-being and resilience, as well as interpersonal family support valued by fasting individuals. ${ }^{12,13}$ These psychosocial factors might have a profound positive interaction with diabetes distress management despite the behavioral challenges experienced during Ramadan. ${ }^{32}$ The present study suggests that the psychosocial benefits of Ramadan observance for individuals with type 2 diabetes should be acknowledged. A second point to note is that incorporation of the FAST tool did not enhance diabetes distress compared to the control group. This is an encouraging observation because individuals using the FAST tool to make informed decisions during Ramadan were not burdened by additional responsibilities such as active self-awareness and management of hypoglycemia as well as dose titration. Thus, the use of the FAST tool to enhance selfmanagement can improve diabetes distress. ${ }^{33}$

There are several limitations to the present study. As with other studies incorporating patient-reported outcomes, recall bias in terms of hypoglycemia reporting cannot be excluded. Corrective actions taken by participants when faced with a hypoglycemia event were also not assessed. However, we incorporated an objective cutoff blood glucose level of $<72 \mathrm{mg} / \mathrm{dL}$ for the hypoglycemia definition to sieve out actual hypoglycemia events. In addition, this was an open-label trial. Whereas the study investigators were reminded to follow the study protocol and use the FAST tool only for intervention participants, the benefits of the FAST tool might have been compromised, owing to a dilution of effect size. However, we nonetheless observed a significantly greater decrease in $\mathrm{HbA}_{1 \mathrm{c}}$ level in the intervention group. Finally, differences among health care clinicians in their practice experience and style might have confounded the study outcome. To minimize this potential limitation, all health care clinicians were briefed and trained on the controlling blood glucose level and was safer compared to the current nonstandardized care practice for managing Muslim individuals with type 2 diabetes who fast during Ramadan. In addition, use of the FAST tool resulted in sustained improvement of FBG level even 3 months after Ramadan. The use of the FAST tool also did not incur additional burden in terms of diabetes distress.

To read or post commentaries in response to this article, see it online at http://www.AnnFamMed.org/content/18/2/139.

Submitted July 13, 2019; submitted, revised, October 15, 2019; accepted October 21, 2019.

Key words: algorithm; diabetes distress; efficacy; fasting blood glucose; $\mathrm{HbA}_{1 \mathrm{c}}$ Muslims; postprandial glucose; Ramadan; safety

Acknowledgments: We would like to acknowledge the coinvestigators from Tan Tock Seng Hospital, including Dr Seow Cherng Jye, Dr Lee Ying Shan, Dr Kon Yin Chian Winston, Dr Quek Peng Lim Timothy, Dr Lim Su Ping Brenda, Dr Tan Wai Kit Alvin, Dr Ho Wai Han, Dr Tan Seng Kiong, Dr Yeo Pei Shan, Dr Stanley Lam, Dr Chin Han Xin, and Ms Chu Shen Onn, for their clinical and administrative contributions to this study. We would also like to acknowledge the coinvestigators from National University Polyclinics, including Dr Wong Sze Mun Cynthia, Dr Taiju Rangpa, Dr Khaw Mei Lin, Ms Bek Siew Joo Esther, and Ms Nur'Ain Bte Abdul Manan, for their clinical and administrative contributions to this study.

Funding support: This clinical trial was supported by an Academic Research Fund Tier 1 grant from the Ministry of Education, Singapore, awarded to Joyce Yu-Chia Lee. The funder was not involved in the design and conduct of the study; collection, management, analysis, and interpretation of the data; preparation, review, and approval of the manuscript; or the decision to submit the manuscript for publication.

Supplemental materials: Available at http://www.AnnFamMed. org/content/18/2/139/suppl/DC1/.

\section{References}

1. Marrero DG, Ard J, Delamater AM, et al. Twenty-first century behavioral medicine: a context for empowering clinicians and patients with diabetes: a consensus report. Diabetes Care. 2013; 36(2):463-470. 
2. Deci EL, Ryan RM. Handbook of Self-Determination Research. Rochester, NY: The University of Rochester Press; 2002.

3. Sallis JF, Owen N, Fisher EB. Ecological models of health behaviour. In: Glanz K, Rimer BK, eds. Health Behaviour and Health Education: Theory, Research, and Practice. 4th ed. San Francisco, CA: JosseyBass; 2008:465-482.

4. Zainudin SB, Ang DY, Soh AW. Knowledge of diabetes mellitus and safe practices during Ramadan fasting among Muslim patients with diabetes mellitus in Singapore. Singapore Med J. 2017;58(5): 246-252.

5. Hassanein M, Al-Arouj M, Hamdy O, et al; International Diabetes Federation (IDF), in collaboration with the Diabetes and Ramadan (DAR) International Alliance. Diabetes and Ramadan: practical guidelines. Diabetes Res Clin Pract. 2017;126:303-316.

6. Bahammam AS, Alaseem AM, Alzakri AA, Sharif MM. The effects of Ramadan fasting on sleep patterns and daytime sleepiness: an objective assessment. J Res Med Sci. 2013;18(2):127-131.

7. Salti I, Bénard E, Detournay B, et al; EPIDIAR Study Group. A population-based study of diabetes and its characteristics during the fasting month of Ramadan in 13 countries: results of the epidemiology of diabetes and Ramadan 1422/2001 (EPIDIAR) study. Diabetes Care. 2004;27(10):2306-2311.

8. Siaw MYL, Chew DE, Toh MP, et al. Metabolic parameters in type 2 diabetic patients with varying degrees of glycemic control during Ramadan: an observational study. J Diabetes Investig. 2016;7(1):70-75.

9. Almansour HA, Chaar B, Saini B. Fasting, diabetes, and optimizing health outcomes for Ramadan observers: a literature review. Diabetes Ther. 2017;8(2):227-249.

10. Ibrahim M, Abu Al Magd M, Annabi FA, et al. Recommendations for management of diabetes during Ramadan: update 2015. BMJ Open Diabetes Res Care. 2015;3(1):e000108.

11. Babineaux SM, Toaima D, Boye KS, et al. Multi-country retrospective observational study of the management and outcomes of patients with Type 2 diabetes during Ramadan in 2010 (CREED). Diabet Med. 2015;32(6):819-828.

12. Al Sifri S, Rizvi K. Filling the knowledge gap in diabetes management during Ramadan: the evolving role of trial evidence. Diabetes Ther. 2016;7(2):221-240.

13. Almansour HA, Chaar B, Saini B. Perspectives and experiences of patients with type 2 diabetes observing the Ramadan fast. Ethn Health. 2018;23(4):380-396.

14. Weinger K. Psychosocial issues and self-care. Am J Nurs. 2007;107(6 Suppl):34-38.

15. Berry E, Lockhart S, Davies M, Lindsay JR, Dempster M. Diabetes distress: understanding the hidden struggles of living with diabetes and exploring intervention strategies. Postgrad Med J. 2015; 91(1075):278-283.

16. Chew BH, Shariff-Ghazali S, Fernandez A. Psychological aspects of diabetes care: effecting behavioral change in patients. World J Diabetes. 2014;5(6):796-808.

17. Young-Hyman D, de Groot M, Hill-Briggs F, Gonzalez JS, Hood K, Peyrot M. Psychosocial care for people with diabetes: a position statement of the American Diabetes Association. Diabetes Care. 2016;39(12):2126-2140.
18. Lum ZK, See Toh WY, Lim SM, et al. Development of a collaborative algorithm for the management of type 2 diabetes during Ramadan: an anchor on empowerment. Diabetes Technol Ther. 2018; 20(10):698-703.

19. Department of Statistics, Ministry of Trade and Industry, Republic of Singapore. Population trends 2016. https://www.singstat.gov. sg/-/media/files/publications/population/population2016.pdf. Published Sep 2016. Accessed Jan 13, 2020.

20. Nathan DM, Kuenen J, Borg R, Zheng H, Schoenfeld D, Heine RJ; A1c-Derived Average Glucose Study Group. Translating the A1C assay into estimated average glucose values. Diabetes Care. 2008; 31(8):1473-1478.

21. Rohlfing CL, Wiedmeyer HM, Little RR, England JD, Tennill A, Goldstein DE. Defining the relationship between plasma glucose and $\mathrm{HbA}(1 \mathrm{c})$ : analysis of glucose profiles and $\mathrm{HbA}(1 \mathrm{c})$ in the Diabetes Control and Complications Trial. Diabetes Care. 2002;25(2):275-278.

22. Monnier L, Colette C, Owens DR. The application of simple metrics in the assessment of glycaemic variability. Diabetes Metab. 2018; 44(4):313-319.

23. Siaw MY, Chew DE, Dalan R, et al. Evaluating the effect of Ramadan fasting on Muslim patients with diabetes in relation to use of medication and lifestyle patterns: a prospective study. Int J Endocrinol. 2014;2014:308546.

24. Lee JY, Wong S. Development and implementation of signs- and symptoms-based insulin adjustment algorithm. Am J Health Syst Pharm. 2010;67(18):1503-1506.

25. Lee JY, Tsou K, Lim J, Koh F, Ong S, Wong S. "Symptom-based insulin adjustment for glucose normalization" (SIGN) algorithm: a pilot study. Diabetes Technol Ther. 2012;14(12):1145-1148.

26. Venkataraman K, Tan LS, Bautista DC, et al. Psychometric Properties of the Problem Areas in Diabetes (PAID) instrument in Singapore. PLoS One. 2015;10(9):e0136759.

27. Introduction: Standards of Medical Care in Diabetes-2019. Diabetes Care. 2019;42(Suppl 1):S1-S2.

28. Ahmedani MY, Alvi SF, Haque MS, Fawwad A, Basit A. Implementation of Ramadan-specific diabetes management recommendations: a multi-centered prospective study from Pakistan. J Diabetes Metab Disord. 2014;13(1):37.

29. Osborn CY, Fisher JD. Diabetes education: integrating theory, cultural considerations, and individually tailored content. Clin Diabetes. 2008;26(4):148-150.

30. Ahmedani MY, Haque MS, Basit A, Fawwad A, Alvi SF. Ramadan Prospective Diabetes Study: the role of drug dosage and timing alteration, active glucose monitoring and patient education. Diabet Med. 2012;29(6):709-715.

31. McEwen LN, Ibrahim M, Ali NM, et al. Impact of an individualized type 2 diabetes education program on clinical outcomes during Ramadan. BMJ Open Diabetes Res Care. 2015;3(1):e000111.

32. Gonzalez-Zacarias AA, Mavarez-Martinez A, Arias-Morales CE, Stoicea N, Rogers B. Impact of demographic, socioeconomic, and psychological factors on glycemic self-management in adults with type 2 diabetes mellitus. Front Public Health. 2016;4:195.

33. Fisher L, Hessler D, Glasgow RE, et al. REDEEM: a pragmatic trial to reduce diabetes distress. Diabetes Care. 2013;36(9):2551-2558. 